\title{
Integrated Care Pathways in Total Hip and Knee Arthroplasty
}

\begin{abstract}
Integrated Care Pathways in Adult Reconstruction refer to perioperative patient care programs designed to reduce the time to functional recovery after total joint arthroplasty surgery. The main objective of such protocols is to reduce costs and resource utilization without compromising quality of care or patient satisfaction.

Integrated Care Pathways are stepwise multidisciplinary programs combining preoperative preparation, intraoperative care, and postoperative methods that positively influence postoperative recovery: patients, surgeons, anesthesiologists, nurses, physiotherapists and occupational therapists are all active participants. This editorial reviews the state of the art of international "fast-track" total knee and hip protocols highlighting that the optimization of multidisciplinary pathways for joint arthroplasty might reduce morbidity and result in shorter length of stay.
\end{abstract}

Keywords: Total knee arthroplasty, Total hip arthroplasty, Integrated care pathways, Fast track, Hip, Knee
Volume 3 Issue 6 - 2015

\author{
Pier Francesco Indelli, ${ }^{1,2}$ Gennaro Pipino, ${ }^{3}$ \\ Mary M Billstrand,' Angelo Graceffa ${ }^{3}$ \\ 'Department of Orthopaedics and Rehabilitation, University of \\ New Mexico, USA \\ ${ }^{2} \mathrm{New}$ Mexico Veteran Affairs Health Care System (NMVAHCS), \\ USA \\ ${ }^{3}$ Department of Health Sciences, Ludes University, Switzerland
}

Correspondence: Pier Francesco Indelli, University of New Mexico, Department of Orthopaedics and Rehabilitation NMVAHCS, I50I San Pedro Drive SE, Albuquerque, NM, 87I 08 , Tel 408-706-6288, Email indelli@va.gov

Received: October 13, 2015 | Published: December 22, 2015
Abbreviations: ICP, Integrated Care Pathways; LOS, Length of Hospital Stay; TKA, Total Knee Arthroplasty; ER, Enhance Recovery; THA, Total Hip Arthroplasty; LIA, Local Infiltration Analgesia

\section{Introduction}

Integrated Care Pathways (ICP) refer to perioperative patient care programs designed to reduce the time to functional recovery after surgery and the length of hospital stay (LOS) ${ }^{1,2}$ It is common knowledge that the high demand for total joint replacements in North America is steadily increasing: between 1996-1997 and 20062007, total hip and knee procedures performed in Canada increased respectively by $59 \%$ and $140 \% .^{3}$ United States is the worldwide leader for the number of total knee arthroplasty (TKA) per 100,000 inhabitants: $221.5 .{ }^{4}$ This increase in demand is worrisome, especially because of a concomitant slower increase in available resources in many countries, including USA, UK and Canada. This is resulting in an increased emphasis on streamlining services applicable to total hip and knee replacement surgeries. Enhance recovery (ER) programs and "Fast-track" protocols are gaining worldwide popularity in order to reduce costs and resource utilization without compromising quality of care or patient satisfaction.

Integrated Care Pathways (ICPs) have been shown to reduce hospital length of stay. ${ }^{5,6}$ complication rates. ${ }^{7}$ and readmission rates. ${ }^{8,9}$ improve postoperative joint function immediately following surgery. ${ }^{10}$ and at one year follow-up. ${ }^{7}$ improve quality of life. ${ }^{11}$ and aid in cost containment. ${ }^{5,6,9,11}$ for patients undergoing total hip (THA) or knee (TKA) arthroplasty. In general, ICP are stepwise multidisciplinary programs combining preoperative preparation, intraoperative care, and postoperative methods that positively influence postoperative recovery: patients, surgeons, anesthesiologists, nurses, physiotherapists and occupational therapists are all active participants. This multimodal approach is also required because THA and TKA procedures address mainly ageing populations with multiple comorbidities and medical treatments: in fact, according to a recent national cohort report, $32.6 \%$ of the patients undergoing TKA had three comorbidities or more, the most frequent ones being hypertension $(67,8 \%)$, diabetes $(20 \%)$ and obesity $(19,8 \%) .{ }^{12}$ Moreover, effective treatment of perioperative pain was highlighted as the most important factor for early mobilization, shortened hospital stay and early discharge. ${ }^{13}$ The anesthetic technique used intraoperatively may also affect the time to hospital discharge and the postoperative mortality rate. ${ }^{14-17}$

\section{Preoperative care}

\section{Patient selection and education}

Patient characteristics preoperative analysis represents the first fundamental step for any ICP for total hip and knee arthroplasty.

A recent study on 3112 THA/TKAs performed following a "fasttrack" protocol included patients with an average age of 67 years. ${ }^{18}$ Although it is difficult to assess the optimal age for patients placed on a "fast-track" protocol, Pereira et al. ${ }^{19}$ recommended an age $\leq 80$ years to be included in a fast-track program and authors of the current study suggest a patient age $\leq 75$ years.

Other patient selection criteria include a $\mathrm{BMI} \leq 35$, adequate home support, adequate functional strength in the upper extremities, absence of history of deep venous thrombosis or pulmonary embolism in the previous 6 months, normal hematocrit, absence of rheumatoid arthritis, no preoperative warfarin and suitable home layout/design. ${ }^{6,7,12,18,19} \mathrm{An}$ American Society of Anesthesiology Score (ASA) $\leq 3$ with no major cardiac or respiratory illness is recommended by many studies..$^{19,20}$

Preoperative education is mandatory in managing patient expectations. Information that is given in a sensitive manner with multidisciplinary support is associated with a reduction in anxiety, hospital LOS and pain in the immediate postoperative period.$^{21-24}$ Preoperative education can be in the form of a group. ${ }^{25}$ a one-toone session with nurse specialists. ${ }^{26}$ or providing an informational DVD. ${ }^{20}$ It should entail a detailed perioperative care plan and set goals and expectations, including a goal for the discharge date. Unfortunately, more than $50 \%$ of patients demonstrate significantly higher expectations of postoperative pain relief, function, and wellbeing than their surgeons. ${ }^{24}$ 


\section{Prehabilitation}

The validity of therapeutic exercises in patients awaiting primary total joint replacement has not been fully demonstrated. Theoretically, improved preoperative functional capacity may improve final outcome. In a recent systematic review, Hoogeboom et al. ${ }^{27}$ reported no beneficial effects of preoperative therapeutic exercises on postoperative functional recovery. On the other hand, Gill et al. ${ }^{28}$ performed a meta-analysis of randomized controlled trials showing that exercise-based interventions can reduce pain and improve physical function for patients awaiting hip replacement surgery but not knee replacement surgery.

\section{Nutrition}

Poor nutritional status affects postoperative recovery and moderate to high-risk patients should be assessed by a dietician prior to THA and TKA. In fact, low serum transferrin levels, low serum albumin and low total lymphocyte count correlate with resource consumption, wound healing, LOS and operative time in patients undergoing joint replacement surgery. ${ }^{29,30}$ According to these findings, oral nutritional supplements should be given to complement patients' diet for a few weeks in the perioperative period. Calorie loading 48 hours before surgery, irrespective of any co-morbidities such as diabetes or high BMI, has been suggested to minimize the adverse metabolic effects of starvation, to reduce the risk of anesthetic and starvation induced diabetes and to maintain high energy levels following the surgical intervention. ${ }^{25}$ This practice might contradict the traditional fasting regimen of six hours aimed at reducing the incidence of pulmonary aspiration, which has minimal scientific support ${ }^{31}$ Recent recommendations suggest fluid intake until 2 hours before the induction of anesthesia and a 6-hour solid food fast. This reduces preoperative thirst, hunger, anxiety, and postoperative insulin resistance. ${ }^{32}$ Preoperative oral intake of carbohydrates also reduces postoperative nausea and vomiting, which hinder postoperative recovery. ${ }^{33} \mathrm{~A}$ preoperative nutritional consult for all patients undergoing total joint reconstruction is standard of care at the author's Institution.

\section{Premedication}

Postoperative pain strongly correlates with slower rehabilitation progression and prolonged hospital LOS..$^{34,35}$ Effective postoperative pain management increases patient satisfaction, improves postoperative outcomes and allows early patient discharge reducing the cost of care. As focus is increasingly directed toward controlling pain and reducing narcotic use, preemptive multimodal analgesia (PMMA) may become standard of care in the perioperative total joint arthroplasty period. In PMMA protocols patients take analgesics preoperatively (preemptive) to prevent central sensitization and amplification of postoperative pain and combine multiple classes of pharmacological agents to maximize analgesia with the fewest side effects..$^{36-38}$ A few meta-analysis of randomized controlled trials showed that preemptive analgesia leads to a decrease in postoperative pain, less total analgesic consumption and overall improved patient comfort. ${ }^{36,39}$ Pre-emptive analgesics used in primary hip and knee joint surgery include gabapentin, nonsteroidal anti-inflammatory drugs (NSAIDS) and cyclooxygenase-2 (COX-2) inhibitors. ${ }^{40,41}$

Gabapentin is a structural analogue of gamma aminobutyric acid and it has been historically used as an antiepileptic drug for partial seizures: an increasing number of studies have suggested that gabapentin used as a preemptive analgesic has a beneficial effect on both pain scores and postoperative opioid consumption after major orthopaedic surgery, including THA and TKA. ${ }^{42-44}$ It is believed that preoperative dosing of gabapentin, between $300 \mathrm{mg}$ and $1200 \mathrm{mg}$ a day, exerts its analgesic effects by preemptively decreasing the spinal cord excitation caused by surgical trauma. ${ }^{45}$

Nonsteroidal anti-inflammatory drugs (NSAIDs) are among the most popular medications used to relieve pain and inflammation and are of particular interest for preemptive analgesia because of their peripheral and central mechanism of action without causing respiratory depression, nausea, sedation or urinary retention. NSAIDs inhibit prostaglandins production, decreasing inflammation, vasodilatation, capillary permeability, pain and fever. The NSAID medications most used as preemptive analgesic are ketoprofen, ibuprofen and acetaminophen. ${ }^{46,47}$ At the author's Institution, patients receive a dose of acetaminophen $1000 \mathrm{mg}$ a day in the three-day preoperative period.

Activation of COX-2 by surgical trauma produces hyperalgesia, which increases the sensitivity of peripheral nociceptors. Several studies indicate that the use of COX-2 inhibitors for preemptive analgesia reduces significantly postoperative pain and, most important, decreases the overall use of opioids after major surgery and even after total joint arthroplasty. ${ }^{47-49}$ Duellman et al. ${ }^{50}$ in an USA based study compared the effect of preemptive analgesia with patient-controlled-analgesia (PCA) in a group of patients undergoing total joint arthroplasties: the patients in the PCA group required significantly more morphine, had a longer average hospital stay and had a significant decrease in participation in postoperative rehabilitation compared with the group that was given a preemptive selective COX-2 inhibitor.

At the author's Institution, a preemptive dose of Celecoxib $200 \mathrm{mg}$ is recommended in the three-day preoperative period in all patients not having specific contraindications detected during the preoperative evaluation. A recent review of perioperative medical management measures. ${ }^{49}$ suggested the use of prophylactic antiemetics, in particular a scopolamine patch, during the perioperative total joint arthroplasty period.

\section{Comparative analgesia techniques}

Total hip and knee arthroplasty can be performed under general or regional anesthesia. A few academic USA institutions are currently increasing the proportions of THA and TKA performed under spinal and/or epidural anesthesia, including Stanford University, the Hospital for Special Surgery, Duke University and the Mayo Clinic. ${ }^{51-54}$ The main advantages of using regional techniques are improved initial postoperative pain relief, more rapid postoperative mobilization, and decreased intraoperative blood loss compared with general anesthesia. ${ }^{55}$ A meta-analysis of randomized clinical trials has found that regional anesthesia reduces postoperative mortality and other complications such as deep vein thrombosis, pulmonary embolism, transfusion requirements, pneumonia, respiratory depression, myocardial infarction and renal failure. ${ }^{56}$ Prolonged epidural analgesia offers advantages over systemic opioid administration by patient controlled analgesia (PCA) and is currently the gold standard in early postoperative pain control after total joint arthroplasty in many European countries. Despite many advantages, there are many concerns of making regional anesthesia the preferred anesthesiologic technique for total joint arthroplasty in the USA. In fact, there is a risk of vascular injury with subsequent bleeding associated with spinal/epidural needle and catheter placement or removal. The development of epidural/spinal hematomas with possible neural compression after neuraxial anesthesia is a special concern in patients receiving anticoagulants. In a recent USA based consecutive series of 100,027 THA and TKA, Pumberger et al. ${ }^{54}$ reported a rate of symptomatic compressive lesions in 1:12,500 of patients undergoing 
spinal and/or epidural anesthesia. According to the American Society of Regional Anesthesia guidelines, non-ASA antiplatelet therapies should be stopped 7 days before surgery and regional anesthesia and cardioprotective ASA should not be discontinued in the preoperative period. ${ }^{57}$ Published data have suggested an increased risk of epidural hematoma development after epidural anesthesia with concomitant administration of Low Molecular weight Heparin (LMWH).$^{58}$

Several regional analgesic techniques exist for perioperative pain management for total joint arthroplasty (TJA). The standard spinal anesthesia technique for THA and TKA often combines intrathecal opioids (i.e. 0,2-0,3 $\mathrm{mg}$ of morphine) and a local anesthetic: intrathecal morphine might also reduce the supplemental postoperative PCA morphine requirements for the patients undergoing THA. ${ }^{59}$ Epidural analgesia with a single injection of extended-release epidural morphine (EREM) or continuous epidural infusions with local anesthetics represent a valid alternative technique, improving pain relief and recovery time after TJA when compared with IVPCA. ${ }^{55,60}$ Peripheral nerve blocks (PNB), including single-injection and continuous PNB catheters (PNBC), have recently received substantial attention as alternatives to neuraxial analgesia. The most used targets for PNB include the femoral nerve, the sciatic nerve and the lumbar plexus. A lumbar plexus block is performed with the goal of anesthetizing additional nerve branches (lateral femoral cutaneous, femoral and obturator nerves) that innervate the THA surgical area. A continuous lumbar plexus block was found to be superior to IV-PCA for pain management, with a reduction in morphine consumption, improvement in pain control, and patient satisfaction. ${ }^{61}$ Although, the lumbar plexus nerve block provides effective analgesia, guidelines from the American Society of Regional Anesthesia and Pain Medicine for patients receiving antithrombotic prophylaxis circumscribe its use..$^{57}$

The most commonly used PNBs for TKA are femoral and sciatic. The knee innervation is mainly covered by the femoral, sciatic, and obturatorius nerves, excepting few areas of the classic incision that are innervated by the lateral cutaneous femoral nerve. In a recent metaanalysis of randomized controlled trials that compare a femoral block with PCA, it has been shown that a femoral block reduced morphine consumption at 24 and 48 hours, pain scores with activity at 24 and 48 hours, and the incidence of nausea. ${ }^{62}$ Salinas et al. ${ }^{63}$ compared a single-injection femoral block to continuous-femoral-nerveblock (CFNB) for TKA: pain intensity ratings were improved and opioids consumption was significantly lower in the forty-eight-hour postoperative period in the CFNB group but there was no difference in hospital LOS or long term functional recovery between the two groups.

Although a femoral block does improve postoperative pain control for TKA, many patients might complain of clinically significant pain because the femoral nerve does not innervate the posterior aspect of the knee. In an attempt to improve pain control after TKA, single and continuous sciatic nerve blocks can be administrated. To date, evidence is mixed for the analgesic benefits of adding a singleinjection or continuous-sciatic-nerve-block (CSNB) to a femoral nerve block for TKA. ${ }^{64} \mathrm{~A}$ recent systematic review established that there is inadequate evidence to define the role of adding a sciatic nerve block for pain control after TKA.$^{65}$ Unlike CFNB, continuous-sciaticnerve-blocks (CSNB) have not been shown to improve functional outcomes or decrease time until discharge readiness. ${ }^{66}$

Adductor canal block is a sensory blockade that provides analgesia in the territory of obturatorius nerve and saphenous nerve. Adductor canal block showed better quadriceps muscle postoperative strength with respect to the FNB and therefore may promote an earlier ambulation without a significant difference in postoperative pain. ${ }^{67-69}$

Among local anesthetics, the most often used long acting local anesthetic for the femoral nerve block is ropivacaine which theoretically provides less motor blockade then bupivacaine. Unfortunately, a good quality femoral block may be associated with weakness of the muscle and risk of fall during the postoperative rehabilitation period. ${ }^{70}$

Factors that must be incorporated into decision making for anesthetic and analgesic technique for total joint arthroplasty include complications and adverse effects. All regional anesthetic techniques have risks, including bleeding, nerve damage, vascular injury, block failure and infection. Neuraxial techniques and deep peripheral nerve catheters have been associated with rare but devastating bleeding complications. Chelly and Schilling documented the safety of placing a peripheral nerve block before the administration of anticoagulation prophylaxis in patients undergoing total joint arthroplasty.$^{70}$ Furthermore, the catheters can be safely removed while the patient is receiving pharmacological prophylaxis after surgery.

A major potential hazard of regional anesthesia techniques, especially CFNB and CSNB, is the association between lowerextremity blocks and patient falls. ${ }^{71-73}$ Continuous peripheral nerve blocks are associated with muscle weakness that may lead to falls or that can delay rehabilitation: special postoperative protocols should be in place to safely manage postoperative quadriceps weakness when patient received CFNB or CSNB.

A modern anesthetic technique for primary THA or TKA should be based on a multimodal approach. Options include:

1. Systemic pain medications that work on different parts of the pain pathway like nonsteroidal anti-inflammatory agents, opioids, and gabapentin.

\section{Peripheral nerve blocks with local anesthetic.}

3. Epidural/spinal anesthesia.

4. Local anesthetic infiltration at the surgical site.

At the author's Institution, the preferred anesthesia protocol for primary THA and TKA include a spinal administration of local anesthetics and opioids plus a single-injection FNB (TKA).

\section{Intraoperative care}

Intraoperative care measures in Integrated Care Pathways for THA and TKA are different from standard care models especially in prevention of blood loss, in the management of surgical drains and in the use of "local infiltration analgesia" (LIA) techniques.

Anemia and hypovolaemia, especially if combined with opioid overuse, may induce postoperative nausea, vomiting, dizziness and orthostatic hypotension leading to an overall slower postoperative recovery. Antifibrinolytics, such as tranexamic acid, can reduce perioperative blood loss in primary total joint arthroplasty ${ }^{74,75}$ The mechanism of action of tranexamic acid is clot breakdown inhibition by reversibly binding both plasminogen and plasmin, preventing cleaving of fibrin and maintaining clot organization. ${ }^{76}$ When tranexamic acid $(10-150 \mathrm{mg} / \mathrm{kg})$ is used during total joint arthroplasty, perioperative blood loss is significantly reduced, as is the need for allogenic blood transfusion .77 and fortunately, the rates of venous thromboembolism are similar to those of controls. ${ }^{76}$ Because of its fibrinolitic but not pro-thrombotic pharmacological effect, the authors of the current editorial recommend an intraoperative (20 minutes 
before tourniquet release in TKA) intravenous dose of $15 \mathrm{mg} / \mathrm{kg}$ of tranexamic acid in any standard total knee arthroplasty procedure, if not contraindicated by patient cardiovascular status. At the author's institution, a single tranexamic acid dose $(10-150 \mathrm{mg} / \mathrm{kg}$ ) is used (I.V. route) thirty minutes before tourniquet release during standard total knee arthroplasty, if not counter indicated by patient health status.

The use of surgical drains in the perioperative management of total joint arthroplasty is controversial: surgical drains use might reduce wound hematoma, periprosthetic joint infection. ${ }^{78}$ and improve postoperative range of motion. On the other side, a closed drainage system promotes blood loss and subsequent need for blood transfusion. ${ }^{79}$ A recent study. ${ }^{80}$ demonstrated that clamping of drains for 3 hours twice in the postoperative day results in significantly less blood loss in the first 48 hours and reduced the need of blood transfusion by 2.2 times compared with no clamping. At the author's Institution, the use of surgical drain is not standard of care but reserved to specific intraoperative bleeding situations.

Local infiltration analgesia (LIA) for pain control after total joint arthroplasty is recently becoming popular. ${ }^{81}$ this technique requires intraoperative infiltration of a mixture of ropivacaine, ketorolac, and adrenaline in the tissue surrounding the surgical wound to achieve satisfactory analgesia decreasing patient perioperative discomfort. Andersen and Kehlet, in a recent review of randomized controlled studies. ${ }^{82}$ suggested that single-shot LIA is similar to continuous femoral nerve blocks, intrathecal morphine analgesia or epidural analgesia for early postoperative periods. Of note, the use of wound catheters does not bring additional benefits. At the author's Institution, LIA techniques are not routinely use because of a risk of contamination during the procedure.

\section{Postoperative care}

Early mobilization ('same day mobilization") is highly recommended for perioperative care after total joint arthroplasty. ${ }^{83,84}$ including patients sitting on the edge of the bed or in a chair, starting postoperative passive or active knee motion or ambulating up to five feet, dependent upon their tolerance. Early mobilization it is a positive predictor for shorter hospital stay, fewer postoperative complications, lower costs and is associated with reduced rates of deep venous thrombosis and pulmonary embolism. ${ }^{85,86}$ Intermittent pneumatic compression devices (foot or calf) are part of many postoperative enhanced recovery programs..$^{87}$ In a recent Cochrane review. ${ }^{88}$ cold therapy ("Cryotherapy") after total knee arthroplasty was found to reduce blood loss and pain level and improve ROM in the first one to two weeks after surgery without any serious adverse event. Aboveknee and below-knee stockings have been found equally safe in preventing deep venous prophylaxis after TKA and are recommended to be use in fast track protocols. ${ }^{89}$ Jain et al. ${ }^{90}$ found a statistically significant reduction in blood loss without compromising range of motion and without an increase in complications when the knee was maintained at $90^{\circ}$ flexion on a static CPM machine in the two postoperative hours following TKA. At the author's Institution, an immediate postoperative continuous-passive-motion (CPM) device is applied to all TKA patients in the immediate postoperative period in conjunction with an articulated cold therapy device and intermittent pneumatic foot compression. All TKA patients use the cold therapy device for twenty-one days postoperatively. Khan et al. ${ }^{91}$ recommend, as part of enhanced recovery after total joint arthroplasty, starting physiotherapy within 3-5 hours of surgery and continuing twice on each subsequent day until discharge.

Postoperative pain management is fundamental in order to achieve a safe early discharge. Opioids are very effective in controlling postoperative pain, but may result in nausea, vomiting, urinary retention, reduced gastrointestinal motility and severe respiratory depression. ${ }^{92}$

The extension of a multimodal analgesic regimen after discharge has been shown to improve pain scores after total joint arthroplasty. ${ }^{93}$ and to allow early patient discharge. The postoperative addition of celecoxib to the traditional analgesic regimen was associated with less pain at 6 weeks and improved knee function when compared with placebo. ${ }^{94}$ Postoperative gabapentinoids reduce opioid-related adverse effects including postoperative nausea, vomiting and urinary retention allowing for a faster patient discharge. ${ }^{95}$ in addition, pregabalin administration in the two post-operative weeks following primary TKA was associated with a decreased rate of neuropathic pain at 3 and 6 months. ${ }^{96}$ Unfortunately, many patients after total joint arthroplasty still receive only an opioid prescription for pain control. Recent studies. ${ }^{93-96}$ suggest that adding other modalities such as NSAIDs, Cox-2 inhibitors and gabapentinoids might improve overall pain management and possibly diminish opioid-related side effects in the early postoperative period.

McCartney \& Nelligan. ${ }^{97}$ suggested a post-discharge multimodal analgesia regimen for total knee arthroplasty which included acetaminophen $1 \mathrm{~g}$ every 8 hours, gabapentin 100-200 mg every 8 hours and celecoxib $100 \mathrm{mg}$ every 12 hours. The use of opioids (Oxycodone 5-10 mg every 4 hours) was reserved as an as needed supplement.

\section{Patient discharge criteria}

Patients discharge should be related to the achievement of expected general and local milestones. General status includes satisfaction with pain and nausea management, satisfactory intake and output, being afebrile, having clear chest sounds and showing good communication regarding concerns and anxieties. Locally, dressing has to be dry and intact and the skin incision has to be clear without sign of infection. A hydrocolloid and ionic silver surgical dressing, routinely used at the Authors institution, supports wound healing by providing a moist environment and it has, at the same time, a sustained antimicrobial activity.

Patients have to demonstrate an understanding of techniques for safe and independent ADL-self-care performance, appropriate use of assistive devices, how to do deep breathing and ankle pumping exercises, minimal independence with bed mobility and transfer and ability to carry out ADLs with minimal assistance or independently. Finally, patients should be able to ambulate 20 meters with walking aid and to climb stairs with minimal assistance or independently. Timing of hospital discharge should be set after a final occupational therapy session: patients should be instructed on how to dress and bathe themself and how to get on and off a toilet while keeping their new joint in the proper position. The occupational therapist might recommend special equipment at home, such as a raised toilet seat, a long-handled shoehorn, or a sock aide.

\section{Outcomes}

Outcome measurements in the forms of LOS, morbidity/mortality, safety aspects, patient satisfaction and economy among others are essential to monitor outcomes regarding all parameters. Many recent reports are promising. Husted et al. ${ }^{98}$ reported that fast-track protocols showed short LOS with high patient satisfaction and few readmissions within 3 months. Husted et al, in a different study.$^{99}$ analyzed differences between THA and TKA fast-track patients in terms of readmission rates: in their study, $15.6 \%$ of patients following 
TKA were readmitted as opposed to $10.9 \%$ after THA $(\mathrm{p}=0.005)$. Wied et al. ${ }^{100}$ reported a prevalence of Manipulation under anesthesia (MUA) of $5.8 \%$ after fast-track TKA: this complication rate was found to mirror other studies investigating incidence of MUA after more conventional TKA treatment protocols with longer LOS. Another study. ${ }^{101}$ showed that, when patients were mobilized within 4 hours postoperatively and the duration of deep venous thrombosis prophylaxis was short (1-4 days), both mortality as well as DVT/PE incidence were extremely low. Economic point of views also favor fast-track surgery as reviews, meta-analyses, and Cochrane reviews on outcomes following fast-track THA and TKA all have found economic savings compare to more conventional set-ups. ${ }^{102}$

\section{Conclusion}

Integrated care pathways for total joint arthroplasty are achieving worldwide popularity: the impact of combining all perioperative care described in this article might be great, but the contribution of any single intervention to clinical outcomes has still to be determined. Improvement in patient outcomes and reduction in length of stay can be associated with modifications of preoperative nurse-led organizational protocols, use of regional anesthetic techniques, multimodal pain regiments, prevention of nausea and vomiting, postural hypotension postoperative control, early aggressive mobilization and rehabilitation, and joint effusion control. Active engagement of multiple disciplines, both medical and allied health, is fundamental.

\section{Acknowledgments}

None.

\section{Conflicts of interest}

None.

\section{References}

1. Fearon KC, Ljungqvist O, Von Meyenfeldt M, et al. Enhanced recovery after surgery: a consensus review of clinical care for patients undergoing colonic resection. Clin Nutr. 2005;24(3):466-77.

2. Kehlet H, Wilmore DW. Multimodal strategies to improve surgical outcome. Am J Surg. 2002;183(6):630-641.

3. Canadian Institute for Health Information. Hip and Knee Replacements in Canada - Canadian Joint Replacement Registry (CJRR) 2008-2009 Annual Report (Ottawa, Ont: CIHI, 2009). 2009.

4. Pina MF, Ribeiro AI, Santos C. Epidemiology and variability of orthopaedic procedures worldwide. In: Bentley G (Ed.), European Instructional Lectures, Springer, Berlin, Germany. 2011;p.9-19.

5. Brunenberg DE, Van Steyn MJ, Sluimer JC, etal.Jointrecovery programme versus usual care: an economic evaluation of a clinical pathway for joint replacement surgery. Med Care. 2005;43(10):1018-1026.

6. Dowsey MM, Kilgour ML, Santamaria NM, et al. Clinical pathways in hip and knee arthroplasty: a prospective randomised controlled study. Med J Aust. 1999;170(2):59-62.

7. Husni ME, Losina E, Fossel AH, et al. Decreasing medical complications for total kneen arthroplasty: effect of critical pathways on outcomes. BMC Musculoskelet Disord. 2010;11:160.

8. Macario A, Horne M, Goodman S, et al. The effect of a perioperative clinical pathway for knee replacement surgery on hospital costs. Anesth Analg. 1998;86(5):978-984.

9. Scranton PE. The cost effectiveness of streamlined care pathways and product standardization in total knee arthroplasty. $J$ Arthroplast 1999;14(2):182-186.
10. Vanhaecht K, Sermeus W, Tuerlinckx G, et al. Development of a clinical pathway for total knee arthroplasty and the effect on length of stay and in-hospital functional outcome. Acta Orthop Belg. 2005;71(4):439-444.

11. Larsen K, Hansen TB, Thomsen PB, et al. Cost-effectiveness of accelerated perioperative care and rehabilitation after total hip and knee arthroplasty. J Bone Joint Surg Am. 2009;91(4):761-772.

12. Pugely AJ, Martin CT, Gao Y, et al. Comorbidities in patients undergoing total knee arthroplasty: do they influence hospital costs and length of stay? Clin Orthop Relat Res. 2014;472(12):3943-3950.

13. Kehlet H, Dahl JB. Anaesthesia, surgery, and challenges in postoperative recovery. The Lancet, Lancet. 2003;362(9399):1921-1928.

14. Vamvakas EC, Carven JH. RBC transfusion and postoperative length of stay in the hospital or the intensive care unit among patients undergoing coronary artery bypass graft surgery: the effects of confounding factors. Transfusion. 2000;40(7):832-839.

15. Ghali WA, Hall RE, Ash AS, et al. Identifying pre- and postoperative predictors of cost and length of stay for coronary artery bypass surgery. Am J Med Qual. 1999;14(6):248-254.

16. Issa ME, Al-Rashedy M, Ballester P, et al. Predictors of duration of postoperative hospital stay in patients undergoing advanced laparoscopic surgery. Surg Laparosc Endosc Percutan Tech. 2005;15(2):90-93.

17. Gagarine A, Urschel JD, Miller JD, et al. Preoperative and intraoperative factors predictive of length of hospital stay after pulmonary lobectomy. Ann Thorac Cardiovasc Surg. 2003;9:222-225.

18. Jorgensen $\mathrm{CC}$, Kehlet H. Role of patient characteristics for fasttrack hip and knee arthroplasty. British Journal of Anaesthesia. 2013;110(6):972-980.

19. Pereira F, Pollard F, Koen R, et al. Reducing hospital stay following total hip and knee replacement surgery with a dedicated fast track program. Current Orthopaedic Practice. 2015;26(1):36-41.

20. Khan SK, Malviya A, Muller SD, et al. Reduced short-term complications and mortality following Enhanced Recovery primary hip and knee arthroplasty: results from 6000 consecutive procedures. Acta Orthopedica. 2014;85(1):26-31.

21. Bozic KJ, Maselli J, Pekow PS, et al. The influence of procedure volumes and standardization of care on quality and efficiency in total joint replacement surgery. J Bone Joint Surg Am. 2010;92:2643-2652.

22. Larsen K, Hvass KE, Hansen TB, et al. Effectiveness of accelerated perioperative care and rehabilitation intervention compared to current intervention after hip and knee arthroplasty. A before-after trial of 247 patients with a 3-month follow-up. BMC Musculoskelet Disord. 2008;9:59.

23. Lee A, Gin T. Educating patients about anaesthesia: effect of various modes on patients' knowledge, anxiety and satisfaction. Curr Opin Anaesthesiol. 2005;18:205-208.

24. Ghomrawi HM, Franco Ferrando N, Mandl LA, et al. How often are patient and surgeon recovery expectations for total joint arthroplasty aligned? Results of a pilot study. HSS J. 2011;7(3):229-34.

25. Dwyer AJ, Thomas W, Humphry S, et al. Enhanced recovery programme for total knee replacement to reduce the length of hospital stay. J Orthop Surg (Hong Kong). 2014;22(2):150-154.

26. Mertens CS, Raut S, Khanduja V. Integrated care pathways in lowerlimb arthroplasty: are they effective in reducing length of hospital stay? Inte Orthop. 2013;37(6):1157-1163.

27. Hoogeboom TJ, Oosting E, Vriezekolk JE, et al. Therapeutic validity and effectiveness of preoperative exercise on functional recovery after joint replacement: a systematic review and meta-analysis. PLoS One. 7(5):e38031. 
28. Gill SD, McBurney H. Does exercise reduce pain and improve physical function before hip or knee replacement surgery? A systematic review and meta-analysis of randomized clinical trials. Arch Phys Med Rehabil. 2013;94(1):164-176.

29. Gherini S, Vaughn BK, Lombardi AV, et al. Delayed wound healing and nutritional deficiencies after total hip arthroplasty. Clin Orthop Relat Res. 1993;293:188-195.

30. Lavernia CJ, Sierra RJ, Baerga L. Nutritional parameters and short term outcome in arthroplasty. J Am Coll Nutr. 1999;18(3):274-278.

31. Ljungqvist $\mathrm{O}$, Soreide E. Preoperative fasting. $\mathrm{Br} J$ Surg. 2003;90:400-406.

32. Thorell A, Efendic S, Gutniak M, et al. Insulin resistance after abdominal surgery. Br J Surg. 1994;81(1):59-63.

33. Hausel J, Nygren J, Thorell A, et al. Randomized clinical trial of the effects of oral preoperative carbohydrates on postoperative nausea and vomiting after laparoscopic cholecystectomy. Br J Surg. 2005;92(4):415-421.

34. Ayalon $\mathrm{O}$, Liu S, Flics $\mathrm{S}$, et al. A multimodal clinical pathway can reduce length of stay after total knee arthroplasty. HSS J. 2011;7(1):9-15.

35. Bottros J, Klika AK, Milidonis MK, et al. A rapid recovery program after total hip arthroplasty. Curr Orthop Pract. 2010;21(4):381-384.

36. Penprase B, Brunetto E, Dahmani E, et al. The efficacy or preemptive analgesia for postoperative pain control: a systematic review of the literature. AORN. 2015;101(1):94-105.

37. Fischer HB, Simanski CJ, Sharp C, et al. A procedure-specific systematic review and consensus recommendations for postoperative analgesia following total knee arthroplasty. Anaesthesia. 2008;63(10):1105-1123.

38. Skinnier HB, Shintani EY. Results of multimodal analgesic trial involving patients with total hip or knee arthroplasty. Am Journal Orthop. 2004;33(2):85-92.

39. Ong CK, Lirk P, Seymour RA, et al. The efficacy of preemptive analgesia for acute postoperative pain management: a meta-analysis. Anest Analg. 2005;100(3):757-773.

40. Feng Y, Ju H, Yang B, et al. Effects of a selective cyclooxygenese-2 inhibitor on postoperative inflammatory reaction and pain after total knee replacement. J Pain. 2008;9(1):45-52.

41. Schroer WC, Diesfeld PJ, LeMarr AR, et al. Benefits of prolonged postoperative cyclooxygenese-2 inhibitor administration on total knee arthroplasty recovery: a double-blind, placebo-controlled study. $J$ Arthroplasty. 2011;26(6 suppl):2-7.

42. Pandey CK, Sahay S, Gupta D, et al. Preemptive gabapentin decreases postoperative pain after lumbar discectomy. Can J Anaesth. 2004;51(10):986-989.

43. Ayalon O, Liu S, Flics S, et al. A multimodal clinical pathway can reduce length of stay after total knee arthroplasty. HSS J. 2011;7(1):9-15.

44. Raphael M, Jaeger M, van Vlymen J. Easily adoptable total joint arthroplasty program allows discharge home in two days. Can J Anaesth. 2011;58:902-910.

45. Moore A, Costello J, Wieczorek MG, et al. Gabapentin improves postcesarean delivery pain management: a randomized, placebo-controlled trial. Anesth Analg. 2011;112(1):167-173.

46. Jung YS, King MK, Um YJ, et al. The effects on postoperative oral surgery pain by varying NSAID administration times: comparison on effect of preemptive analgesia. Oral Surg Oral Med Oral Pathol Oral Radiol Endod. 2005;100(5):559-563.

47. Kashefi P, Honarmand A, Safavi M. Effects of preemptive analgesia with colecoxib and acetaminophen on postoperative pain relef following lower extremity orthopaedic surgery. Adv Biomed Res. 2012;1:66-72.
48. Dorr LD, Raya J, Long WT, et al. Multimodal analgesia without parental narcotics for TKA. J Arthroplasty. 2008;23(4):502-508.

49. Baldini A, Caldora P. Perioperative Medical Management for Total Joint Arthroplasty. Springer International Publishing, Switzerland. 2015.

50. Duellman TJ, Gaffigan C, Milbrandt JC, et al. Multi-modal, preemptive analgesia decreases the length of hospital stay following total joint arthroplasty. Orthopedics 2009. 2009;32(3):167-173.

51. Hebl JR, Lennon RL. Mayo Clinic Atlas of Regional Anesthesia and Ultrasound-Guided Nerve Blockade. Mayo Clinic Scientific Press. 2010;pp.488.

52. Indelli PF, Vail TP, Grant S, et al. Regional anaesthesia in major hip surgery. Clin Orthop. 2005;441:250-255.

53. Brodsky JB, Mariano ER. Regional anaesthesia in the obese patient: lost landmarks and evolving ultrasound guidance. Best Practice and Research Clinical Anaesth. 2011;25(1):61-72.

54. Pumberger M, Memtsoudis SG, Stundner O, et al. An analysis of the safety of epidural and spinal neuraxial anesthesia in more than 100.000 consecutive major lower extremity joint replacements. Reg Anesth Pain Med. 2013;38(6):515-519.

55. Choi PT, Bhandari M, Scott J, et al. Epidural analgesia for pain relief following hip and knee replacement. Cochrane Database Syst review. 2003;3:CD003071.

56. Rodgers A, Walker N, Schung S, et al. Reduction of postoperative mortality and morbidity with epidural or spinal anaesthesia: results from overview of randomized clinical trials. BMJ. 2000;321(7275):1493.

57. Horlocker TT, Wedel DJ, Rowlingson JC, et al. Regional anesthesia in the patient receiving antithrombotic or thrombolytic therapy: American Society of regional Anesthesia and Pain Medicine Evidence-Based Guidelines (Third edn). Reg Anesth Pain Med. 2010;35(1):64-101.

58. Wysowski DK, Talarico L, Bacsanyi J, et al. Spinal and epidural hematoma and low-molecular-weight-heparin. $N$ Engl J Med. 1998;338(24):1774-1775.

59. Rathmell JP, Pino CA, Taylor R, et al. Intrathecal morphine for postoperative analgesia: a randomized, controlled, dose ranging study after hip and knee arthroplasty. Anesth Analg. 2003;97(5):1452-1457.

60. Hartrick CT, Martin G, Kantor G, et al. Evaluation of a single dose, extended-release epidural morphine formulation for pain after knee arthroplasty. J Bone Joint Surg Am. 2006;88(2):273-281.

61. Siddiqui ZI, Cepeda MS, Denman W, et al. Continuous lumbar plexus block provides improved analgesia with fewer side effects compared with systemic opioid after hip arthroplasty: a randomized-controlled trial. Reg Anesth Pain Med. 2007;32(5):393-398.

62. Paul JE, Arya A, Hurlburt L, et al. Femoral nerve block improves analgesia outcomes after total knee arthroplasty: a meta-analysis of randomized controlled trials. Anesthesiology. 2010;113(5):1144-1162.

63. Salinas FV, Liu SS, Mulroy MF. The effect of single-injection femoral nerve block versus continuous femoral nerve block after total knee arthroplasty on hospital stay and long-term functional recovery with an established clinical pathway. Anesth Analg. 2006;102(4):1234-1239.

64. Ilfeld BM, Madison SJ. The sciatic nerve and knee arthroplasty: to block, or not to block-that is the question. Reg anesth Pain Med. 2011;36(5):421-423.

65. Abdallah FW, Brull R. Is sciatic nerve block advantageous when combined with femoral nerve block for postoperative analgesia following total knee arthroplasty? A systematic review. Reg Anesth Pain Med. 2011;36(5):493-498.

66. Wegener JT, van Ooij B, van Dijk CN, et al. Value of single-incision or continuous sciatic nerve block in addition to a continuous femoral nerve block in patients undergoing total knee arthroplasty: a prospective, randomized, controlled trial. Reg Anesth Pain Med. 2011;36(5):481-488. 
67. Mudumbai SC, Kim TE, Howard SK, et al. Continuous adductor canal blocks are superior to continuous femoral nerve blocks in promoting early ambulation after TKA, Clinical Orthopaedics and Related Research. 2014;472(5):1377-1383.

68. Jæger P, Zaric D, Fomsgaard JS, et al. Adductor canal block versus femoral nerve block for analgesia after total knee arthroplasty: a randomized, double-blind study. Regional Anesthesia and Pain Medicine. 2013;38(6):526-532.

69. Bonica JJ. Current status of postoperative pain therapy. In: Dubnet R (Ed.), Current Topics in Pain Research and Therapy, Tokyo, Japan. 1983;pp.169-189.

70. Chelly JE, Schilling D. Thromboprophylaxis and peripheral nerve blocks in patients undergoing total joint arthroplasty. $J$ Arthroplasty. 2008;23(3):350-354.

71. Ilfeld BM, Duke KB, Donohue MC. The association between lower extremity continuous peripheral nerve blocks and patient falls after knee and hip arthroplasty. Anesthesia \& Analgesia. 2010;111(6):1552-1554.

72. Kandasami M, Kinninmonth AW, Sarungi M, et al. Femoral nerve block for total knee replacement - a word of caution. Knee. 2009;16(2):98-100.

73. Sharma S, Iorio R, Specht LM, et al. Complication of femoral nerve block for total knee arthroplasty. Clin Orthop Relat Res. 2010;468(1):135-140.

74. Orpen NM, Little C, Walker G, et al. Tranexamic acid reduces early post-operative blood loss after total knee arthroplasty: a prospective randomised controlled trial of 29 patients. Knee. 2006;13(2):106-110.

75. Zhang H, Chen J, Chen F, et al. The effect of tranexamic acid on blood loss and use of blood products in total knee arthroplasty: a metaanalysis. Knee Surg Sports Traumatol Arthrosc. 2012;20(9):1742-1752.

76. Gillette BP, DeSimone LJ, Trousdale RT, et al. Low risk of thromboembolic complications with tranexamic acid after primary total hip and knee arthroplasty. Clin Orthop Relat Res. 2013;471(1):150-154.

77. Yang ZG, Chen WP, Wu LD. Effectiveness and safety of tranexamic acid in reducing blood loss in total knee arthroplasty: a meta-analysis. $J$ Bone Joint Surg Am. 2012;94(13):1153-1159.

78. Volpe L, Indelli PF, Latella L, et al. Periprosthetic Joint Infections (PJI): A Clinical Practice Algorithm. Joints. 2014;2(4):169-174.

79. Parker MJ, Livingstone V, Clifton R, et al. Closed suction surgical wound drainage after orthopaedic surgery. Cochrane Database Syst Rev. 2007;18(3):CD001825.

80. Pornrattanamaneewong C, Narkbunnam R, Siriwattanasakul P, et al. Three-hour interval drain clamping reduces postoperative bleeding in total knee arthroplasty: a prospective randomized controlled trial. Arch Orthop Trauma Surg. 2012;132(7):1059-1063.

81. Kerr DR, Kohan L. Local infiltration analgesia: a technique for the control of acute postoperative pain following knee and hip surgery-a case study of 325 patients. Acta Orthop. 2008;79(2):174-183.

82. Andersen LO, Kehlet H. Analgesic efficacy of local infiltration analgesia in hip and knee arthroplasty: a systematic review. British Journal of Anaesthesia. 2014;pp.1-15.

83. Bottros J, Klika AK, Milidonis MK, et al. A rapid recovery program after total hip arthroplasty. Curr Orthop Pract. 2010;21(4):381-384.

84. Gulotta LV, Padgett DE, Sculco TP, et al. Fast Track THR: One Hospital's Experience with a 2-Day Length of Stay Protocol for Total Hip Replacement. HSS J. 2011;7(3):223-228.

85. Khan F, Ng L, Gonzalez S, et al. Multidisciplinary rehabilitation programmes following joint replacement at the hip and knee in chronic arthropathy. Cochrane Database Syst Rev. 2008;2:CD004957.
86. Mont MA, Jacobs JJ, Boggio LN, et al. Preventing venous thromboembolic disease in patients undergoing elective hip and knee arthroplasty. J Am Acad Orthop Surg. 2011;19(12):768-776.

87. Stowers MDJ, Lemanu DP, Coleman B, et al. Review Article: Perioperative care in enhanced recovery for total hip and knee arthroplasty. JOS. 2014;22(3):383-392.

88. Adie S, Kwan A, Naylor JM, et al. Cryotherapy following total knee replacement. Cochrane Database of Systematic Reviews. 2012;9:CD007911.

89. Williams LA, Owen TD. Above-knee versus beloe-knee stockings in total knee arthroplasty. Ann R Coll Surg Engl. 2006;88(3):302-305.

90. S Jain, A Dinah, S Palmer. Knee flexion significantly reduces blood loss and transfusion rate after uncemented total knee arthroplasty. The Internet Journal of Orthopedic Surgery. 2008;11(1).

91. Khan SK, Malviya A, Muller SD, et al. Reduced short-term complications and mortality following Enhanced Recovery primary hip and knee arthroplasty: results from 6000 consecutive procedures. Acta Orthopaedica. 2014;85(1):26-31.

92. Zargar-Shoshtari K, Hill AG. Optimization of perioperative care for colonic surgery: a review of the evidence. ANZ J Surg. 2008;78(1-2):13-23.

93. Carmichael NM, Katz J, Clarke H, et al. An intensive perioperative regimen of pregabalin and celecoxib reduces pain and improves physical function scores six weeks after total hip arthroplasty: a prospective randomized controlled trial. Pan Resmanag. 2013;18(3):127-132.

94. Schroer WC, Diesfeld PJ, LeMarr AR, et al. Benefits of prolonged postoperative cyclooxygenase-2 inhibitor administration on total knee arthroplasty recovery. J Arthroplasty. 2011;26(Suppl 6):2-7.

95. Tiippana EM, Hamunen K, Kontinen VK, et al. Do surgical patients benefit from perioperative gabapentin/pregabalin? A systematic review of efficacy and safety. Anesth Analg. 2007;104(6):1545-1556.

96. Buvanendran A, Kroin JS, Della Valle CJ, et al. Perioperative oral gabapentin reduces chronic pain after total knee arthroplasty: a prospective, randomized, controlled trial. Anesth Analg. 2010;110:199-207.

97. McCartney CJL, Nelligan K. Postoperative pain management after total knee arthroplasty in elderly patients: treatment options. Drugs Aging. 2014;31(2):83-91.

98. Husted H, Holm G, Jacobsen S. Predictors of length of stay and patient satisfaction after hip and knee replacement surgery: fast-track experience in 712 patients. Acta Orthop. 2008;79(2):168-173.

99. Husted H, Otte KS, Kristensen BB, et al. Readmissions after fast-track hip and knee arthroplasty. Arch Orthop Trauma Surg. 2010;130(9):1185-1191.

100. Wied C, Thomsen MG, Kallemose T, et al. The risk of manipulation under anesthesia due to unsatisfactory knee flexion after fast-track total knee arthroplasty. Knee. 2015;22(5):419-423.

101. Husted H, Kristensen BB, Otte KS, et al. Low incidence of thromboembolic complications in a fast-track set up with hip and knee arthroplasty. Acta Orthop. 2010;81(5):599-605.

102. Husted H. Fast-track hip and knee arthroplasty: clinical and organizational aspects. Acta Orthopaedica. 2012;83(346):1-39. 